\title{
Perfil socioeconômico, clínico e farmacoterapia de pacientes geriátricos com Diabetes
}

\author{
Mellitus \\ Socioeconomic, clinical and pharmacotherapy profile of geriatric patients with Diabetes Mellitus \\ Perfil socioeconómico, clínico y farmacoterapéutico de pacientes geriátricos con Diabetes Mellitus
}

Recebido: 15/02/2021 | Revisado: 22/02/2021 | Aceito: 25/02/2021 | Publicado: 04/03/2021

Cristina Silva Pereira
ORCID: https://orcid.org/0000-0001-8099-5156
Universidade José do Rosário Vellano, Brasil
E-mail: kristinasp27@gmail.com
Isadora Gonçalves Lino
ORCID: https://orcid.org/0000-0002-9689-4664
Universidade José do Rosário Vellano, Brasil
E-mail: isahglino27@gmail.com
Pamela de Jesus Barbosa
ORCID: https://orcid.org/0000-0003-1235-0545
Universidade José do Rosário Vellano, Brasil
E-mail: pamela.barbosa17@gmail.com
Victória Toledo Silva
ORCID: https://orcid.org/0000-0003-0056-4108
Universidade José do Rosário Vellano, Brasil
E-mail: victoria.silva@aluno.unifenas.br
Roberta Bessa Veloso Silva
ORCID: https://orcid.org/0000-0003-4794-5872
Universidade José do Rosário Vellano, Brasil
E-mail: roberta.veloso@unifenas.br
Gérsika Bitencourt Santos
ORCID: https://orcid.org/0000-0003-0849-2786
Universidade José do Rosário Vellano, Brasil
E-mail: gersika.santos@unifenas.br

\section{Resumo}

No Brasil, há estudos que comprovam o quanto os quadros epidemiológicos de doenças crônicas, como o diabetes mellitus, vêm crescendo, afetando diretamente os pacientes geriátricos. O presente estudo teve como objetivo analisar o perfil socioeconômico e clínico, bem como a farmacoterapia dos pacientes geriátricos que apresentam diabetes mellitus. Esta é uma pesquisa descritiva e quantitativa, na qual foram analisadas variáveis relacionadas ao o perfil socioeconômico, clínico e a terapêutica dos pacientes idosos. Os dados foram representados por meio de tabelas e análise estatística. Os resultados mostraram que a obesidade grau 1 ocorreu com maior frequência em idosos que recebem de 1 a 2 salários mínimos, com diabetes tipo 2 e do gênero feminino. Observou-se que em pacientes com sobrepeso, também prevaleceu o diabetes tipo 2, porém houve uma predominância do gênero masculino. Deve-se destacar que quase em sua totalidade, os idosos declararam ausência da prática de exercícios físicos. O medicamento oral mais utilizado foi a Metformina e dos injetáveis foi a insulina NPH, seguido da insulina regular. Em relação à realização de exames para o controle glicêmico verificou-se que geralmente estes são feitos anualmente.

Palavras-chave: Diabetes mellitus; Farmacoterapia; Antidiabéticos.

\begin{abstract}
In Brazil, there are studies that prove how much the epidemiological conditions of chronic diseases, such as diabetes mellitus, are growing more and more, directly affecting geriatric patients. The present study aimed to analyze the socioeconomic and clinical profile, as well as the pharmacotherapy of geriatric patients with diabetes mellitus. This is a descriptive and quantitative research, in which variables related to the socioeconomic, clinical and therapeutic profile of elderly patients were analyzed. The data were represented using tables and statistical analysis. The results showed that grade 1 obesity occurred more frequently in the elderly who receive 1 to 2 minimum wages, with type 2 diabetes and females. It was observed that in overweight patients, type 2 diabetes also prevailed, but there was a predominance of males. It should be noted that almost entirely, the elderly declared an absence of physical activity. The most widely used oral medication was Metformin and the injectables were NPH insulin, followed by regular insulin. Regarding tests for glycemic control, it was found that this is usually done annually.
\end{abstract}

Keywords: Diabetes mellitus; Pharmacological treatment; Antidiabetics. 


\begin{abstract}
Resumen
En Brasil, hay estudios que demuestran cuánto crecen cada vez más las condiciones epidemiológicas de las enfermedades crónicas, como la diabetes mellitus, afectando directamente a los pacientes geriátricos. El presente estudio tuvo como objetivo analizar el perfil socioeconómico y clínico, así como la farmacoterapia de pacientes geriátricos con diabetes mellitus. Se trata de una investigación descriptiva y cuantitativa, en la que se analizaron variables relacionadas con el perfil socioeconómico, clínico y terapéutico de los pacientes ancianos. Los datos se representaron mediante tablas y análisis estadístico. Los resultados mostraron que la obesidad de grado 1 se presentó con mayor frecuencia en los ancianos que reciben de 1 a 2 salarios mínimos, con diabetes tipo 2 y en mujeres. Se observó que en los pacientes con sobrepeso también predominó la diabetes tipo 2, pero hubo predominio del sexo masculino. Cabe señalar que, casi en su totalidad, los ancianos declararon ausencia de actividad física. El medicamento oral más utilizado fue la metformina y los inyectables fueron la insulina NPH, seguida de la insulina regular. En cuanto a las pruebas de control glucémico, se encontró que estes suele realizarse anualmente.
\end{abstract}

Palabras clave: Diabetes mellitus; Farmacoterapia; Antidiabéticos.

\title{
1. Introdução
}

O envelhecimento é um processo natural e inerente ao homem. Apesar de não ser sinônimo de invalidez ou enfermidade, é comum que na velhice a saúde esteja fragilizada, sendo a maioria dos idosos polimedicados (Cruz, Beltrame, Dallacosta, 2019; Salles, 2019). Este processo é caracterizado por alterações moleculares e celulares, que levam à diminuição da homeostasia e, como consequência, tem-se a apoptose celular, envolvendo alterações morfofuncionais, bioquímicas, fisiológicas e psicológicas, o que proporciona o surgimento de doenças crônicas e degenerativas, visto que inúmeros sistemas perdem sua funcionalidade (Junior, 2019). O diabetes mellitus é uma das doenças crônicas mais comuns em idosos, atingindo cerca de 23,5\% dos brasileiros na faixa etária igual ou superior a 65 anos (Santos et al., 2019).

Enquanto o diabetes mellitus tipo 1 (DM1) é caracterizado como uma doença autoimune que afeta as células beta pancreáticas, diminuindo a produção de insulina, o diabetes mellitus tipo 2 (DM2) é caracterizado como uma resistência periférica à insulina. O DM2 está relacionado à senescência, devido às mudanças metabólicas, celulares e à inatividade física. (Shakeri et al., 2018; Kalyani, Golden, Cefalu, 2017). Caracterizam-se como distúrbios metabólicos causadores de hiperglicemia persistente, a qual é associada a complicações micro e macrovasculares, além de reduzir a qualidade e a expectativa de vida, pois aumenta a taxa de mortalidade (Sociedade Brasileira de Diabetes, 2017). O excesso de glicose no sangue aumenta os níveis de estresse oxidativo, estando intimamente relacionado a outras patologias, como a nefropatia diabética, aterosclerose e cardiomiopatias (Kalyani, Golden, Cefalu, 2017).

Para diagnosticar o diabetes mellitus, os exames mais recomendados, segundo as Diretrizes da Sociedade Brasileira de Diabetes (2017-2018) são: glicemia em jejum, teste oral para tolerância à glicose e hemoglobina glicada (HbA1c). A prevenção se faz necessária, a fim de diminuir o avanço da doença. A educação em saúde é a maneira mais positiva para o controle glicêmico, de forma a incentivar alimentação saudável e a prática de exercícios físicos com consequente controle do peso, visto que a obesidade está intrinsicamente ligada ao diabetes (Lima et al., 2020; Ramos et al., 2017).

O controle da glicemia e do peso do paciente se faz importante na prevenção de complicações decorrentes do diabetes, como o Acidente Vascular Cerebral (AVC), aterosclerose e nefropatias, as quais contribuem para a diminuição da qualidade de vida e o aumento da mortalidade (Sociedade Brasileira de Diabetes, 2017-2018). Nesse sentido, este trabalho teve como objetivo analisar o perfil socioeconômico e clínico, bem como farmacoterapia de pacientes geriátricos que apresentam diabetes mellitus.

\section{Metodologia}

Foi desenvolvida uma pesquisa descritiva e quantitativa. Foram incluídos na pesquisa 58 pacientes diabéticos de ambos os sexos, com idade superior a 60 anos, atendidos em uma Unidade Básica de Saúde (UBS) de uma cidade do sul de Minas Gerais. Os pacientes foram selecionados de acordo com a disponibilidade do pesquisador, ou seja, baseado no número de idosos 
diabéticos residentes no distrito da cidade considerada. Apesar da impossibilidade de generalização de resultados, uma amostra não-probabilística pode ser útil e, até mesmo, preferível em relação a amostra probabilística em uma série de situações. O importante é que suas limitações estejam claras para que não haja erros na análise dos resultados (Ferreira, 2018).

Os dados foram coletados no período de janeiro a março de 2020, por meio de entrevistas aos pacientes realizadas durante as consultas agendadas e por análise do prontuário eletrônico ESUS. Para a realização desta pesquisa, foram utilizados formulários para a coleta de dados com questões que foram pré-elaboradas. As variáveis coletadas foram socioeconômicas e clínicas, tais como o tipo de diabetes, o tempo da doença, a farmacoterapia utilizada, o estilo de vida (dieta e exercícios físicos) e o Índice de Massa Corporal (IMC).

Este trabalho foi aprovado pelo comitê de ética em pesquisa da Universidade José do Rosário Vellano sob parecer número 3.953.496.

A organização e apresentação dos dados foram feitas por meio de tabelas. O teste qui-quadrado foi utilizado para elaborar os intervalos de confiança para proporção (95\%) para as todas as variáveis, quer sejam faixa etária, gênero, escolaridade, renda familiar, entre outras, e para checar a hipótese de independência entre o tipo de diabetes e o tipo de medicamento, a prática de exercícios físicos e o IMC.

\section{Resultados e Discussão}

O diabetes mellitus é um distúrbio metabólico, crônico, manifestado pelo aumento dos níveis de glicose no sangue (hiperglicemia) e que limita diretamente na qualidade de vida dos pacientes (Campos et al., 2016). O diagnóstico da patologia diabetes mellitus se torna mais comum com o avanço da idade. O diagnóstico da doença se acentua a partir dos 45 anos, e mais de um quarto dos indivíduos com 65 anos ou mais referem terem diabetes (Francisco et al., 2018). Observou-se que 35 (60,34\%) dos 58 pacientes diabéticos deste estudo tinham entre 60 e 70 anos de idade, $14(24,14 \%)$ entre 71 e 80 anos e 9 (15,52\%) acima de 80 anos. Deve-se destacar que houve a predominância de diabetes mellitus em pacientes do gênero feminino, 35 (60,34\%), quando comparado a $23(39,65 \%)$ do gênero masculino (Tabela 1$)$.

Há muitos aspectos que favorecem a adesão ao tratamento, como a escolaridade, a renda salarial e a idade dos indivíduos, e estes interferem diretamente na maneira que o paciente irá aderir à terapêutica no seu dia a dia (Campos et al., 2016). O grau de escolaridade mais frequente entre os pacientes foi o ensino fundamental incompleto, 23 (39,65\%), seguido de $15(25,86 \%)$ com ensino médio incompleto; 8 (13,79\%) com ensino fundamental completo; 7 (12,07\%) com ensino médio completo e $3(5,17 \%)$ com ensino superior incompleto. Pode-se verificar que a renda familiar dos pacientes entrevistados se apresenta baixa, sendo que destes, 91\% vivem com apenas 1 salário mínimo, o que afeta diretamente a maneira em que o tratamento farmacológico será selecionado (Tabela 1). 
Tabela 1 - Dados sociodemográficos obtidos de 58 pacientes idosos com diabetes mellitus e seus respectivos valores-p e intervalos de confiança (95\%).

\begin{tabular}{|c|c|c|c|}
\hline Variáveis & $n(\%)$ & Valor-p & IC $(95 \%)$ \\
\hline \multicolumn{4}{|l|}{ Faixa etária (anos) } \\
\hline $60-70$ anos & $35(60,34 \%)$ & $0,1486 n s$ & $46,64-72,67$ \\
\hline 71-80 anos & $14(24,14 \%)$ & $0,0001^{* *}$ & $14,27-37,46$ \\
\hline Mais de 80 anos & $9(15,52 \%)$ & $<0,01 * *$ & $0,08-0,28$ \\
\hline \multicolumn{4}{|l|}{ Gênero } \\
\hline Feminino & $35(60,34 \%)$ & $0,1486 n s$ & $46,64-72,67$ \\
\hline Masculino & $23(39,65 \%)$ & $0,1486 n s$ & $0,27-0,53$ \\
\hline \multicolumn{4}{|l|}{ Escolaridade } \\
\hline Ensino Fundamental & $23(39,65 \%)$ & $0,1486 n s$ & $0,27-0,53$ \\
\hline \multicolumn{4}{|l|}{ Incompleto } \\
\hline Ensino Fundamental & $8(13,79 \%)$ & $<0,01^{* *}$ & $6,56-25,93$ \\
\hline \multicolumn{4}{|l|}{ Completo } \\
\hline Ensino Médio & $15(25,86 \%)$ & $0,0004^{* *}$ & $15,65-39,29$ \\
\hline \multicolumn{4}{|l|}{ incompleto } \\
\hline Ensino Médio completo & $7(12,07 \%)$ & $<0,01^{* *}$ & $5,39-23,91$ \\
\hline Ensino Superior & $3(5,17 \%)$ & $<0,01^{* *}$ & $1,34-15,30$ \\
\hline \multicolumn{4}{|l|}{ incompleto } \\
\hline Ensino Superior & $2(3,45 \%)$ & $<0,01^{* *}$ & $0,60-12,95$ \\
\hline \multicolumn{4}{|l|}{ completo } \\
\hline \multicolumn{4}{|l|}{ Renda familiar } \\
\hline 1 a 2 salários mínimos & $53(91,38 \%)$ & $<0,01^{* *}$ & $80,28-96,78$ \\
\hline 3 a 5 salários mínimos & $4(6,90 \%)$ & $<0,01^{* *}$ & $2,23-17,55$ \\
\hline 6 a 10 salários mínimos & $1(1,72 \%)$ & $<0,01^{* *}$ & $0,00-10,46$ \\
\hline Mais de 10 & $0(0,00 \%)$ & $<0,01^{* *}$ & $0,00-7,74$ \\
\hline salários mínimos & & & \\
\hline
\end{tabular}

\footnotetext{
${ }^{* *}$ Significativo ao nível nominal de $1 \%$ de significância $(\mathrm{p}<0,01)$.

${ }^{n s}$ Não significativo ao nível nominal de $5 \%$ de significância ( $\left.\mathrm{p}>0,05\right)$.

Fonte: Autores.
}

Perante os dados levantados, ficou destacado que 40 (68,96\%) indivíduos apresentavam diabetes mellitus tipo 2 , em contrapartida, $15(25,86 \%)$ apresentavam diabetes mellitus tipo 1 e 3 pacientes $(5,17 \%)$ apresentavam outros tipos de diabetes, sendo que dentre estes que relataram apresentar outros tipos de diabetes, $2 \%$ a $3 \%$ obtiveram diabetes mellitus após o uso de algumas medicações ou durante o período gestacional (Tabela 2). Dos idosos pesquisados, 24 (41,38\%) pacientes descobriram a patologia próximo dos seus 35 anos, 15 (25,86\%) já sabiam desde a infância e 16 (27,56\%) não souberam nos informar há quanto tempo apresentavam a patologia estudada.

O diabetes mellitus tipo 2 é o mais frequente, acometendo indivíduos acima de 40 anos e pode estar associado a fatores de risco modificáveis, como sobrepeso ou obesidade, dislipidemia, disfunção endotelial, hipertensão arterial sistêmica (HAS), e 
a fatores não modificáveis, como fatores genéticos e a idade dos indivíduos. Já o diabetes mellitus tipo 1 (DM1) caracteriza-se como uma patologia autoimune causada pela destruição das células beta-pancreáticas, responsáveis pela produção de insulina. Ao longo da vida, o indivíduo necessita de administração do hormônio via exógena para a redução da hiperglicemia (Rossaneis et al., 2019).

Portanto, a secreção de insulina é irregular nesses pacientes, sendo insuficiente para compensar a resistência à insulina. Essa resistência à insulina pode ser revertida com a redução de peso, exercícios físicos e /ou tratamento farmacológico de hiperglicemia, mas é extremamente raro a glicemia deste paciente retornar ao normal. O risco de desenvolver o diabetes mellitus tipo 2 aumenta com a idade, obesidade e falta de exercício físico (Moretto et al., 2016).

Um fator importante pesquisado durante o estudo foi a prática de exercícios físicos que os pacientes exerciam. Apenas $5(8,62 \%)$ relataram praticá-los. Registrou-se que $53(91,38 \%)$ pacientes não praticavam exercícios físicos (Tabela 2). Dentre os pacientes que argumentaram realizar exercícios, 80\% afirmaram realizá-los geralmente entre 1 a 3 vezes da semana e $20 \%$ praticavam diariamente; estes informaram praticar exercícios físicos ao ar livre, como caminhadas e outros tipos de atividades aeróbicas.

Os exercícios físicos são bastante úteis no tratamento e reabilitação de várias disfunções crônico-degenerativas, dentre elas a diabetes mellitus. Sendo assim, o tratamento do diabético não deve ficar restrito apenas ao profissional médico, mas deve ser feito por uma equipe multiprofissional, não se baseando apenas em medicamentos, pois como toda doença crônica, o diabetes implica em uma mudança de estilo de vida. Portanto, um programa de exercícios só deve ser iniciado após o afastamento de situações que predisponham o agravamento das complicações inerentes ao diabético (Carvalho, Silva \& Coelho, 2015).

Diante dos dados levantados, observou-se que 44,83\% dos pacientes realizavam seus exames em tempo anual. Apenas 6,9\% dos entrevistados realizavam seus exames a cada 2 meses e 32,76\% a cada 6 meses; no entanto, muitos não realizavam o exame na frequência adequada, sendo que $15,52 \%$ dos pacientes alegaram não realizar os exames com frequência ou não lembravam da data, o que pode estar interferindo diretamente para que não se atinja o controle glicêmico ideal.

Tabela 2 - Condições de saúde e estilo de vida de 58 pacientes idosos com DM.

\begin{tabular}{|c|c|c|c|}
\hline Variáveis & $n(\%)$ & Valor-p & IC95(\%) \\
\hline \multicolumn{4}{|l|}{ Tipo de diabetes } \\
\hline Tipo 1 & $15(25,86 \%)$ & $0,0004^{* *}$ & $15,65-39,29$ \\
\hline Tipo 2 & $40(68,96 \%)$ & $0,0058^{* *}$ & $55,31-80,10$ \\
\hline Outro & $3(5,17 \%)$ & $<0,01^{* *}$ & $1,34-15,30$ \\
\hline \multicolumn{4}{|l|}{ Diagnóstico } \\
\hline Infância & $15(25,86 \%)$ & $0,0004^{* *}$ & $15,65-36,29$ \\
\hline Após 35 anos & $24(41,38 \%)$ & $0,2373 n s$ & $28,86-55,04$ \\
\hline \multicolumn{3}{|l|}{ de medicamentos } & $0,60-12,95$ \\
\hline Após gravidez & $1(1,72 \%)$ & $<0,01^{* *}$ & $0,00-10,46$ \\
\hline Não sabem & $16(27,59 \%)$ & $0,0010^{* *}$ & $17,05-41,11$ \\
\hline \multicolumn{4}{|l|}{ IMC } \\
\hline Abaixo do normal & $3(5,17 \%)$ & $<0,01^{* *}$ & $1,34-15,30$ \\
\hline Normal & $9(15,52 \%)$ & $<0,01^{* *}$ & $7,77-27,92$ \\
\hline Sobrepeso & $12(20,69 \%)$ & $<0,01^{* *}$ & $11,59-33,72$ \\
\hline
\end{tabular}




Obesidade grau 1
Obesidade grau 2
Obesidade grau 3
Exercício físico
Sim
Não

Exame laboratorial

$\begin{array}{lllr}\text { A cada } 2 \text { meses } & 4(6,90 \%) & <0,01^{* *} & 2,23-17,55 \\ \text { A cada } 6 \text { meses } & 19(32,76 \%) & 0,0126^{*} & 21,35-46,46 \\ \text { A cada } 12 \text { meses } & 26(44,83 \%) & 0,5115 n s & 31,96-58,37 \\ \text { Não realiza } & 9(15,52 \%) & <0,01^{* *} & 7,77-27,92\end{array}$$$
53(91,38 \%)
$$

$<0,01^{* *}$

$<0,01^{* *}$

$3,22-19,72$

$80,28-96,78$

$$
\begin{aligned}
& 27(46,55 \%) \\
& 2(3,45 \%) \\
& 5(8,62 \%)
\end{aligned}
$$

$\begin{array}{lr}0,6936 \mathrm{~ns}^{*} & 33,54-60,02 \\ <0,01^{* *} & 0,60-12,95 \\ <0,01^{* *} & 3,22-19,72\end{array}$

com frequência

Antidiabéticos

\section{injetáveis}

Insulina Lispro/Aspart

Insulina Regular

$0(0,00 \%)$

Insulina NPH

$19(32,76 \%)$

$37(63,79 \%)$

Insulina Ultra

Lenta (Glargina)

Liraglutida

$2(3,45 \%)$

\section{Antidiabéticos orais}

Glibenclamida

$6(10,34 \%)$

Glimeperida

$4(6,90 \%)$

Glicazida

Metformina

$3(5,17 \%)$

Dapaglifozina+

Metfomina

Vildagliptina+

$40(68,96 \%)$

$2(3,45 \%)$

$3(5,17 \%)$
$<0,01^{* *}$

$0,0126^{*}$

$0,0489^{*}$

$<0,01^{* *}$

$0,0345^{*}$

$0,60-12,95$

Metformina

\footnotetext{
${ }^{* * *}$ Significativo ao nível nominal de $1 \%$ de significância $(\mathrm{p}<0,01)$.

* Significativo ao nível nominal de $5 \%$ de significância $(\mathrm{p}<0,05)$.

${ }^{n s}$ Não significativo ao nível nominal de $5 \%$ de significância (p>0,05).

Fonte: Autores.
}

\begin{tabular}{|c|c|c|c|c|}
\hline \multicolumn{3}{|c|}{ Variáveis } & \multirow[b]{2}{*}{$\operatorname{IC95}(\%)$} & \multirow[b]{2}{*}{ Valor-p } \\
\hline Variáveis & \multicolumn{2}{|c|}{ Diabetes mellitus $n(\%)$} & & \\
\hline Medicamento & DM 1 & DM 2 & & \\
\hline Oral & $8(38,09 \%)$ & $28(60,87 \%)$ & $-0,51 ; 0,06$ & $0,1415 n s$ \\
\hline
\end{tabular}

Tabela 3 - Correlação entre o tipo de diabetes e o IMC, tipo de diabetes e a prática de exercícios físicos, bem como a renda familiar e IMC, renda familiar e o nível de escolaridade, com seus respectivos intervalos de confiança (95\%) e valores-p. 
Injetável
Ambos

Valor-p

IMC

Normal

Sobrepeso

Obesidade grau 1

Obesidade grau 2

Obesidade grau 3

Valor-p

\section{IMC (feminino)}

$\begin{array}{ll}\text { Normal } & 2(40,00 \%) \\ \text { Sobrepeso } & 2(40,00 \%) \\ \text { Obesidade grau 1 } & 1(20,00 \%) \\ \text { Obesidade grau 2 } & 0(0,00 \%) \\ \text { Obesidade grau 3 } & 0(0,00 \%) \\ \text { Valor-p } & \mathbf{0 , 0 8 8 9} \text { ns }\end{array}$

\section{IMC (masculino)}

$\begin{array}{ll}\text { Normal } & 1(16,67 \%) \\ \text { Sobrepeso } & 3(50,00 \%) \\ \text { Obesidade grau 1 } & 2(33,33 \%) \\ \text { Obesidade grau 2 } & 0(0,00 \%) \\ \text { Obesidade grau 3 } & 0(0,00 \%) \\ \text { Valor-p } & \mathbf{0 , 2 5 7 7 ~} \mathbf{n s}\end{array}$

\section{Exercício físico}

$\begin{array}{ll}\text { Sim } & 3(20,00 \%) \\ \text { Não } & 12(80,00 \%)\end{array}$

Valor-p

$0,1010 n s$
$0(0,00 \%)$

$18(39,13 \%)$

$-0,06 ; 0,51$

$0,1415 n s$

$\begin{array}{lll}5(13,16 \%) & -0,20 ; 0,48 & 0,5143 n s \\ 7(18,42 \%) & -0,11 ; 0,65 & 0,1504 n s \\ 21(55,26 \%) & -0,65 ; 0,09 & 0,1960 n s \\ 0(0,00 \%) & - & - \\ 5(13,16 \%) & -0,30 ; 0,03 & 0,4814 n s\end{array}$

$\begin{array}{lll}5(20,00 \%) & -0,38 ; 0,77 & 0,6994 n s \\ 3(12,00 \%) & -0,29 ; 0,85 & 0,3808 n s \\ 16(64,00 \%) & -0,96 ; 0,08 & 0,1874 n s \\ 0(0,00 \%) & - & - \\ 1(4,00 \%) & -0,16 ; 0,08 & 1,0000 n s\end{array}$

$0(0,00 \%) \quad-0,25 ; 0,59 \quad 0,6839 n s$ $4(30,77 \%) \quad-0,40 ; 0,79 \quad 0,7671 n s$ $5(38,46 \%) \quad-0,56 ; 0,46 \quad 1,0000 n s$ $0(0,00 \%)$

$4(30,77 \%)$

$-0,68 ; 0,06$

$0,3555 n s$

\section{Renda familiar}

\section{Escolaridade}

Fund. incompleto

Fund. completo

Médio incompleto

Médio completo
1 a 2 (sal. mín.)

$19(35,85 \%)$

$8(15,09 \%)$

$14(26,41 \%)$

$6(11,32 \%)$
3 a 4 (sal. mín.)

$\begin{array}{lll}0(0,00 \%) & 0,09 ; 0,62 & 0,3593 n s \\ 0(0,00 \%) & -0,08 ; 0,38 & 0,9270 n s \\ 1(25,00 \%) & -0,44 ; 0,47 & 1,0000 n s \\ 1(25,00 \%) & -0,70,0,46 & 0,9889 n s\end{array}$




$\begin{array}{lllll}\text { Superior incompleto } & 2(3,77 \%) & 1(25,00 \%) & -0,77 ; 0,35 & 0,5015 \mathrm{~ns} \\ \text { Superior completo } & 0(0,00 \%) & 1(25,00 \%) & -0,81 ; 0,31 & 0,0896 \mathrm{~ns} \\ \text { Nenhum } & 4(7,55 \%) & 0(0,00 \%) & -0,07 ; 0,22 & 1,0000 \mathrm{~ns} \\ \text { Valor-p } & \mathbf{0 , 0 1 8 0 ~ n s} & & & \end{array}$

\section{IMC}

$\begin{array}{lll}\text { Normal } & 7(13,21 \%) & 1 \\ \text { Sobrepeso } & 11(20,74 \%) & 2 \\ \text { Obesidade grau 1 } & 25(47,17 \%) & 1 \\ \text { Obesidade grau 2 } & 2(3,77 \%) & 0 \\ \text { Obesidade grau 3 } & 5(9,43 \%) & 0 \\ \text { Abaixo do peso } & 3(5,66 \%) & 0 \\ \text { Valor-p } & \mathbf{0 , 6 5 8 6 ~ n s} & \\ \text { icativo ao nível nominal de 1\% de significância }(\mathrm{p}<0,01) . \\ \text { cativo ao nível nominal de 5\% de significância }(\mathrm{p}<0,05) . \\ \text { gnificativo ao nível nominal de 5\% de significância }(\mathrm{p}>0,05) .\end{array}$

\footnotetext{
** Significativo ao nível nominal de $1 \%$ de significância $(\mathrm{p}<0,01)$.

* Significativo ao nível nominal de $5 \%$ de significância $(\mathrm{p}<0,05)$.

${ }^{n} s$ Não significativo ao nível nominal de $5 \%$ de significância $(\mathrm{p}>0,05)$

Fonte: Autores
}

$\begin{array}{lll}1(25,00 \%) & -0,67 ; 0,43 & 1,0000 n s \\ 2(50,00 \%) & -0,93 ; 0,34 & 0,4677 n s \\ 1(25,00 \%) & -0,36 ; 0,80 & 0,7354 n s \\ 0(0,00 \%) & -0,05 ; 0,13 & 1,0000 n s \\ 0(0,00 \%) & -0,08 ; 0,27 & 1,0000 n s \\ 0(0,00 \%) & -0,06 ; 0,17 & 1,0000 n s\end{array}$

Em relação ao diabetes mellitus, a hiperglicemia persistentemente prolongada é maléfica ao organismo do indivíduo. Há uma estreita relação entre níveis elevados de glicose no sangue e a manifestação das complicações do diabetes. O desequilíbrio inalterável acarreta, durante o passar dos anos, uma série de complicações orgânicas, o que afetará diretamente os tecidos, gerando lesões e perda de funções, podendo chegar até a falência de vários órgãos (Rossaneis et al., 2019).

Entre os medicamentos orais mais destacados nos dados coletados estavam Metformina, 40 (68,96\%), Glicazida, 3 $(5,17 \%)$, Glibenclamida, $6(10,34 \%)$ e Glimeperida, 4 (6,90\%). Já entre os antidiabéticos injetáveis mais utilizados pela população estudada estavam a Insulina NPH, 37 (63,79\%), e a Insulina Regular, 19 (32,76\%) (Tabela 2).

Logo, diante dos dados levantados, $40(68,96 \%)$ indivíduos entrevistados realizavam a administração da Metformina, um medicamento que apresenta um baixo custo e que os pacientes obtinham facilmente. Em relação aos medicamentos injetáveis, apenas 3\% dos entrevistados faziam o uso de Liraglutida. Segundo a IDF (2017), as diretrizes apontam que a metformina é considerado como fármaco de primeira escolha para o início do tratamento farmacológico dos indivíduos que apresentam diabetes mellitus do tipo 2, com concentrações de $500 \mathrm{mg}$ a $2000 \mathrm{mg}$ por dia, sendo administrado junto ou após as refeições (IDF, 2017).

Os medicamentos Vildagliptina + Metformina e Dapaglifozina + Metformina não eram utilizados com frequência entre os pacientes entrevistados, 4\% dos pacientes utilizavam a Dapaglifozina + Metformina e 3\% utilizavam a Vildagliptina + Metformina (Tabela 2). Estes possuem um alto custo, sendo de difícil acesso para a grande maioria dos entrevistados, tendo em vista que $53(91,38 \%)$ recebem em torno de 1 salário mínimo.

Meramente em casos em que a farmacoterapia não alcança a solução dos problemas, é feita uma associação entre medicamentos orais ou com insulinoterapia. As escolhas realizadas frente ao tratamento para os indivíduos para controlar a glicemia são bem amplas, abrangendo as mudanças nos hábitos de vida, o que pode ser uma dificuldade para o paciente, além da parte farmacoterapêutica, que implica os fármacos sensibilizadores de insulina (biguanidas e tiazolidinedionas), secretagogos de insulina (glinidas e Sulfonilureias), inibidores da alfa-glicosidase, terapias à base de incretina (Inibidores de DPP-4 e Agonistas do receptor GLP-1), co-transportador de sódio-glicose-2, análogos peptídicos da amilina, insulinas de ação rápida, ação curta, ação intermediária, ação prolongada, pré-misturado e inalado (Da Silva, 2019). 
$\mathrm{Na}$ Tabela 3, verifica-se que em se tratando da escolaridade e a renda familiar, observou-se que elas estão associadas, $(\mathrm{p}=0,0180)$. Dos $53(91,38 \%)$ que ganham entre 1 e 2 salários, 19 (35,85\%) têm ensino fundamental incompleto; 14 (26,41\%) têm ensino médio incompleto; 8 (15,09\%) têm ensino fundamental completo; 6 (11,32\%) têm ensino médio completo; 2 (3,77\%) têm ensino superior incompleto e $4(7,55 \%)$ não tinham ensino.

A compreensão sobre o diabetes é de suma importância para o processo de autocuidado, permitindo impactar a população acerca dos fatores de risco para o desenvolvimento do diabetes, bem como os contratempos que a patologia crônica apresenta, possibilitando a melhor adesão ao cuidado desses tratamentos. Estudos indicam que as pessoas mais bem informadas, mais motivadas e com esquemas de medicação menos complicadas acabam possuindo maior adesão ao tratamento indicado pelo especialista (Campos et al., 2016).

Pode-se observar que apesar da não significância estatística ao se relacionar o tipo de diabetes e o tipo de medicamento ( $\mathrm{p}=0,1415)$, verificou-se que dos $46(68,66 \%)$ pacientes com diabetes tipo 2, $28(60,87 \%)$ utilizaram apenas medicamentos por via oral e $18(39,13 \%)$ utilizaram ambos (oral e injetável). Quanto ao tipo 1, dos 21 (31,34\%), 8 (38,09\%), o fazer por via oral e, $13(61,90 \%)$, fazem a ingestão via oral e injetável, (Tabela 3).

\section{Conclusão}

Os resultados permitiram concluir que a obesidade grau 1 ocorreu com maior frequência em idosos que recebem de 1 a 2 salários mínimos, com diabetes tipo 2 e do gênero feminino. Observou-se que em pacientes com sobrepeso, também prevaleceu o diabetes tipo 2, porém houve uma predominância do gênero masculino. Deve-se destacar que, quase em sua totalidade, os idosos declararam ausência da prática de exercício físico. O medicamento mais utilizado foi a Metformina, por ser de baixo custo, e dos injetáveis foi a insulina NPH, seguida da insulina regular. Em relação à realização de exames para o controle glicêmico, verificou-se que geralmente são feitos anualmente.

\section{Referências}

Campos, T. S. P. et al. (2016). Fatores associados à adesão ao tratamento de pessoas com diabetes mellitus assistidos pela atenção primária de saúde. J. Health Biol Sci, 4(4), 251-256.

Carvalho, S. S., Silva, T. M. de A., \& Coelho, J. M. F. (2015). Contribuições do tratamento não farmacológico para Diabetes Mellitus tipo 2. Revista de Epidemiologia e Controle de Infecção, 5(2), 59-64.

Cruz, R. R., Beltrame, V., \& Dallacosta, F. M. (2019). Envelhecimento e vulnerabilidade: análise de 1062 idosos. Rev. Bras. Geriatr. Gerontol., $22(3)$, e180212.

Da Silva, T. M. (2019). Revisão bibliográfica sobre o diagnóstico e o tratamento do diabetes mellitus. Trabalho de Conclusão de Curso - Monografia. Curso de Bacharelado em Farmácia, Centro de Educação e Saúde, Universidade Federal de Campina Grande, Cuité. Paraíba. Brasil.

Ferreira, D. F. (2018). Estatística multivariada. (3a ed.), UFLA.

Francisco, P. S. B., et al. (2018). Prevalência simultânea de hipertensão e diabetes em idosos brasileiros: desigualdades individuais e contextuais. Ciênc. saúde coletiva, 23(11), 3829-3840.

International Diabetes Federation - IDF (2017). Atlas diabetes mellitus no brasil e no mundo por regiões em 2017 e em 2045 . Brasil.

Junior, E. C. da S. (2019). Mecanismos moleculares envolvidos com o envelhecimento celular e cutâneo (Trabalho de Conclusão de Curso). Centro Universitário de Brasília - UNICEUB. Faculdade de Ciências da Educação e Saúde. Brasília, DF, Brasil.

Kalyani, R. R., Golden, S. H. \& Cefalu, W. T. (2017). Diabetes e envelhecimento: considerações e objetivos únicos de cuidado. Diabetes Care, 40(4), 440-443. Lima, A. P. de, et al. (2020). Conhecimento e atitude sobre o diabetes tipo 2 em idosos: estudo de base populacional. Ciência saúde coletiva, 25(2), 729-740.

Moretto, M. C. et al. (2016). Associação entre cor/raça, obesidade e diabetes em idosos da comunidade: dados do Estudo FIBRA. Cad. Saúde Pública [online], 32(10), e00081315.

Salles, J. (2019). Sociedade Brasileira de Diabetes. Dia Mundial do Idoso Mais exercícios, menos medicamentos: as particularidades do tratamento do diabetes na terceira idade. https://www.diabetes.org.br/publico/component/content/article/212-teste-noticia/1718-dia-mundial-do-idoso-mais-exercicios-menosmedicamentos-as-particularidades-do-tratamento-do-diabetes-na-terceira-idade? Itemid $=445$ 
Research, Society and Development, v. 10, n. 3, e5310312995, 2021

(CC BY 4.0) | ISSN 2525-3409 | DOI: http://dx.doi.org/10.33448/rsd-v10i3.12995

Shakeri, H. et al. (2018). A senescência celular relaciona o envelhecimento e o diabetes nas doenças cardiovasculares. American Journal of Physiology, 315(3), H448-H462.

Sociedade Brasileira de Diabetes - SBD. Diretrizes da Sociedade Brasileira de Diabetes: 2017-2018. Clannad.

Ramos, R. de S. P. da S. et al. (2017). Fatores associados ao diabetes em idosos assistidos em serviço ambulatorial especializado geronto-geriátrico. Rev. Bras. Geriatr. Gerontol., 20(3), 364-374.

Rang, H. P., Dale, M. M., \& Ritter, J. M. (2016). Farmacologia. (8a ed.), Elsevier.

Rossaneis, M. A. et al. (2019). Fatores associados ao controle glicêmico de pessoas com diabetes mellitus. Ciênc. saúde coletiva 24(3), 997-1005.

Santos, A. dos S. et al. (2019). Uso de medicamentos e estilo de vida no gerenciamento do diabetes em idosos. Revista de Salud Pública, 21 (3), e377822.

Da Silva, T. M. (2019). Revisão bibliográfica sobre o diagnóstico e o tratamento do diabetes mellitus. Trabalho de Conclusão de Curso - Monografia. Curso de Bacharelado em Farmácia, Centro de Educação e Saúde, Universidade Federal de Campina Grande, Cuité. Paraíba. Brasil.

Silveira, É. A., Vieira, L. L. \& Souza, J. D. (2018). Elevada prevalência de obesidade abdominal em idosos e associação com diabetes, hipertensão e doenças respiratórias. Ciênc. saúde coletiva 23(3), 903-912. 